\title{
Quaderni
}

QUADERNI Communication, technologies, pouvoir

102 | Hiver 2020-2021

Politique(s) des dystopies

\section{Maquetter une revue : des formes aux positionnements scientifiques et des positionnements aux formes}

Olivier Bertrand et Étienne Candel

\section{(2) OpenEdition Journals \\ Édition électronique \\ URL : http://journals.openedition.org/quaderni/1841 \\ DOI : 10.4000/quaderni.1841 \\ ISSN : 2105-2956 \\ Éditeur \\ Les éditions de la Maison des sciences de l'Homme}

Édition imprimée

Date de publication : 5 janvier 2021

Pagination : 5-8

Référence électronique

Olivier Bertrand et Étienne Candel, « Maquetter une revue : des formes aux positionnements scientifiques et des positionnements aux formes », Quaderni [En ligne], 102 | Hiver 2020-2021, mis en ligne le 05 janvier 2021, consulté le 29 janvier 2021. URL : http://journals.openedition.org/quaderni/ 1841 ; DOI : https://doi.org/10.4000/quaderni.1841 


\section{Maquetter une revue: des formes aux position- nements scientifiques et des positionnements aux formes}

\section{Olivier Bertrand}

Éditeur (Surfaces Utiles) et designer graphique

\section{Étienne Candel}

Co-directeur de Quaderni 
Avec ce numéro 102, Quaderni inaugure sa nouvelle maquette, fruit d'une collaboration entre le comité de rédaction, d'une part, et Olivier Bertrand et Marine Montagné, éditeurs, d'autre part. Inscrite principalement en Science politique et en Sciences de l'information et de la communication, Quaderni interroge la communication au croisement des technologies et du pouvoir. Un changement de "maquette", un changement de la forme éditoriale, c'est, précisément, une transformation ou un déplacement du rapport de la revue à ses objets, de la relation de la revue à ses publics ef au monde dans lequel objets, revue, publics, chercheurs, lecteurs et partenaires évoluent.

Une nouvelle maquette, donc, qui manifeste un déplacement dans la politique de la revue. Depuis la disparition de son fondateur Lucien Sfez, la décision a été prise de poursuivre l'histoire de Quaderni, et de lui conserver son identité scientifique, cette "ligne" éditoriale fondatrice d'un certain regard et d'un certain territoire, interdisciplinaire et ouvert à la multitude des regards qui traversent et interrogent nos champs. Une revue ouverte sur les diverses disciplines des Sciences anthroposociales (pour reprendre le mot de Jean-Michel Berthelot), capable d'accueillir géographes, historiens, anthropologues ou sociologues, juristes, littéraires, philosophes... mais aussi acteurs des mondes politique ou économique. Le comité de rédaction s'ouvre à de nouveaux membres, la direction devient bicéphale et interdisciplinaire', encourageant de fait des formes collaboratives de travail et des formes renouvelées de dialogue avec les chercheurs de son environnement.

Une maquette nouvelle, également, parce que l'objet éditorial et son image, inévitablement, ne sont pas sans rapport avec leur contexte. L'intervention d'Olivier Bertrand et Marine Montagné a été sollicitée d'abord en raison de leur identité en tant qu'éditeurs: leur familiarité avec l'édition scientifique est en effet doublée d'une pratique de l'édition d'art et, plus généralement, d'une expertise en design éditorial. Cette identité est en cohérence avec le projet actuel de Quaderni pour les prochaines années: la revue entend en effet, à la suite de son héritage historique d'ouverture, favoriser le développement de formes renouvelées de la communication scientifique - du format des entretiens aux notes de recherche, du compte rendu d'événements à l'accent placé sur la jeune recherche et le dialogue.

Comment raconter la façon dont l'ambition communicationnelle d'une revue comme Quaderni en vient à s'objectiver dans sa forme? Sensibles aux médiations matérielles de la communication, qui impliquent que tout processus de communication soit interrogé dans son rapport structurant aux techniques, aux technologies et, plus généralement, aux cadres sociaux de son effectuation, nous donnons ici à voir comment les éditeurs et graphistes ont élaboré

1. Emmanuel Taïeb est professeur de Science politique à Sciences Po Lyon, Étienne Candel de Sciences de l'information et de la communication à l'IUT de Paris. 
la mutation du design éditorial de Quaderni afin de rendre la revue visuellement plus attractive et d'en faciliter la reconnaissance en bibliothèque.

\section{AUGMENTER LE CONTRASTE}

Nous choisissons de garder la typographie emblématique de titrage de la revue - la célèbre Avant Garde, une police d'écriture linéale géométrique dessinée par Herb Lubalin au début des années 70 - en préférant un style plus gras (Bold) et en l'assignant au paratexte de la revue (avant-propos, titres courants, folios, etc.). L'objectif de l'opération est d'augmenter significativement la densité du gris typographique de le page en la tachetant d'une certaine "épaisseur" noire qu'offre la fonte Bold.

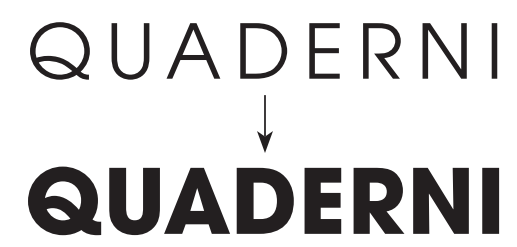

Pour le texte de labeur, nous couplons l'Avant Garde à la Vremena, remplaçant ainsi la Times - un classique typographique signé Stanley Morison - qui était utilisé dans la maquette précédente. Bien que la Vremena partage des proportions communes avec le Times, elle en propose toutefois une actualisation légèrement moins maniérée et davantage contrastée, peut-être plus contemporaine.

\section{Times New Roman Vremena}

\section{AXER/DÉSAXER}

Les colonnes de texte des différentes rubriques s'organisent autour d'un axe central commun à toute la nouvelle maquette. Le Dossier, dans l'axe, est valorisé par le blanc des marges confortables qui l'entourent. Les colonnes du reste des rubriques (Varia, Interview, Livres en revue) dansent autour de cet axe pour se différencier les unes des autres.
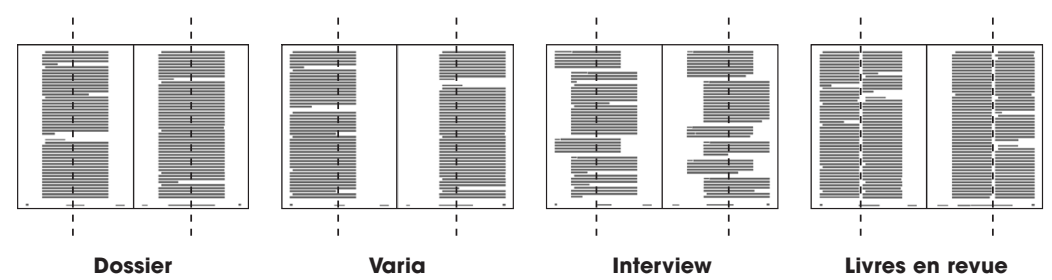


\section{RYTHMER}

Selon l'équation "rythme=rupture" et inspirés par certaines pratiques éditoriales propres à des périodiques moins universitaires, nous ouvrons à l'intérieur des articles des espaces de commentaire (offert aux auteurs) et de mise en exergue (offert aux éditeurs) pour rompre le caractère monotone du texte de labeur. Les commentaires se désaxent de la colonne de texte et les exergues occupent une page entière en grands caractères.

\section{YEUX TYPOGRAPHIQUES}

Pour différencier et identifier la revue, nous misons sur les instincts de chasseur-cueilleur du lecteur en affichant en couverture une paire d'yeux typographiques à reconnaître dans la jungle des tables de librairies ou des rayons de bibliothèques. Élément de fixation et stéréotype de l'œil universitaire qui, derrière ses lunettes, décrypte et analyse.

Suivant l'humeur du numéro de Quaderni, il sera tantôt curieux, tantôt déboussolé, parfois fâché...
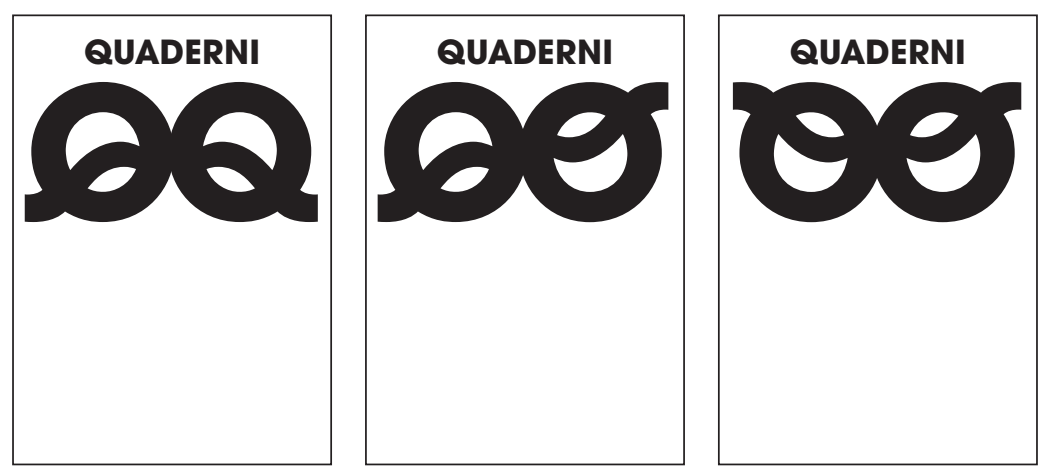

Sans préjudice de ses possibles interprétations facétieuses, le redoublement de l'initiale du nom de la revue appuie avant tout une lecture iconique et travaille la forme en logo, voire en une sorte de mascotte que nos lecteurs trouveront plaisir, nous l'espérons, à voir réapparaitre sous diverses tonalités colorielles et divers régimes indiciaires, de numéro en numéro, au gré des thématiques abordées.

On le perçoit, la nouvelle maquette de Quaderni est liée à un projet - lisible dans ses rubriques, dans les perspectives qu'elle donne pour un renouvellement de l'énonciation auctoriale et éditoriale, dans l'ambition de (re)fonder une voix pleinement polyphonique dans l'univers des revues. Elle est aussi une promesse faite à nos lecteurs de suivre, depuis les formes que nous adoptons jusqu'aux objets que nous prenons en charge, le mouvement et les transformations d'un monde de la communication qu'il nous appartient, en tant que scientifiques, d'observer, de questionner et de donner à comprendre. 